\title{
Three-Dimensional Boundary layer Flow and Heat Transfer of a Fluid Particle Suspension over a Stretching Sheet Embedded in a Porous Medium
}

https://doi.org/10.1515/nleng-2018-0008

Received January 10, 2018; revised October 20, 2018; accepted December 28, 2018.

\begin{abstract}
This article presents the effect of nonlinear thermal radiation on three dimensional flow and heat transfer of fluid particle suspension over a stretching sheet. The combined effects of non-uniform source/sink and convective boundary condition are taken into consideration. The governing partial differential equations are transformed into ordinary differential equations using similarity variables, which are then solved numerically by using Runge Kutta Fehlberg-45 method with shooting technique. The influence of various parameters on velocity and temperature profiles are illustrated graphically, and discussed in detail. The results indicate that the fluid phase velocity is greater than that of the particle phase for various existing parameters.
\end{abstract}

Keywords: Convective boundary condition; Fluid particle suspension; nonlinear thermal radiation; Stretching sheet; Three dimensional flow

\section{Introduction}

Heat transfer phenomenon due to suspended particles into the fluid has important role in recent and advanced processes of industrial and engineering problems concerned with powder technology, sedimentation, rain erosion in guided missiles, combustion, atmospheric fallout, fluidization, nuclear reactor cooling, electrostatic precipi-

H.B. Mallikarjuna, Department of Mathematics, Adichunchanagiri institute of technology, Chickkamgalore, Karnataka, India, E-mail: mallikarjunahb.ait@gmail.com

*Corresponding Author: M.C. Jayaprakash, Department of Information Technology, Al Musanna college of technology, Oman, E-mail: jayaprakashmc@gmail.com

Raghavendra Mishra, Department of Mathematics, Government Post Graduate College, Ranikhet (Almora), Uttarakhand, India, Email: rahavendramisra@yahoo.in tation of dust, waste water treatment, acoustics batch settling and so forth. A study on fundamentals of dusty fluid was made by Saffman [1]. Vajravelu and Nayfeh [2] have investigated the hydromagnetic flow of a dusty fluid over a porous stretching sheet. Some more investigations on heat transfer process with fluid particle suspension can be seen in the Refs [3-9]. Al-Rashed et al. [10] report the influence of surface waviness on natural convection boundary layer flow of the two-phase dusty fluid having compressible nature. Recently the effect of thermal stratification on MHD flow and heat transfer of dusty fluid over a vertical stretching sheet embedded in a thermally stratified porous medium in the presence of uniform heat source and thermal radiation has been numerically investigated by Gireesha et al. [11].

Thermal radiation plays an important role in manufacturing industries for the design of nuclear power plants and several engineering applications. Due to its vital applications, numerous researchers have paid their attention to thermal radiation effect [12-17]. Mahanthesh et al. [18] investigated the Marangoni transport of dissipating SWCNT and MWCNT nanofluids under the influence of magnetic force and radiation. Further, it is worth to notice that the linear radiation is valid for small temperature difference. But, for the larger temperature difference nonlinearized Rosseland approximation is to be considered. Hayat et al. [19] initiated the tangent hyperbolic nanofluid flow in the presence of nonlinear thermal radiation. The idea of nonlinear thermal radiation along with heat transfer phenomenon has recently been presented by so many researchers (see [20-23]). Recently, Prasannakumara et al. [24] studied the effect of nonlinear thermal radiation on slip flow and heat transfer of fluid particle suspension with nanoparticles over a nonlinear stretching sheet immersed in a porous medium.

Three dimensional flow has many applications in solar collectors, aeronautical engineering, science and technology, crude oil purification, magnetic material processing, geophysics and controlling of cooling rate, insulation engineering, grain storage devices, ground water pollution, purification process and petroleum reservoirs. 
Wang [25] proposed three dimensional boundary layer flow induced by a stretching surface. The unsteady laminar boundary-layer flow of a viscous electrically conducting fluid induced by the impulsive stretching of a flat surface in two lateral directions through an otherwise quiescent fluid has been studied by [26] Takhar et al. The three dimensional flow and heat transfer over a stretching surface has been carried out by Ahmad et al. [27]. Ahmad and Nazar [28] studied the hydromagnetic flow and heat transfer over a bidirectional stretching surface. Choudhury and Das [29] studied the viscoelastic effect on free convective three-dimensional flow along with the phenomenon of heat and mass transfer. Nadeem et al. [30] examined the MHD three dimensional Casson fluid flow past a porous linearly stretching sheet. MHD three dimensional flow of couple stress fluid was studied by Ramzan et al. [31].

Convective flow in porous media has been widely studied in the recent years due to its wide applications in engineering as geophysical thermal and insulation engineering, the modeling of packed sphere beds, the cooling of electronic systems, groundwater hydrology, chemical catalytic reactors, ceramic processes, grain storage devices, fiber and granular insulation, petroleum reservoirs, coal combustors, ground water pollution and filtration processes, to name just a few of these applications. Chamkha et al. [32,33] studied the natural convection past an isothermal sphere in a Darcy porous medium saturated with a nanofluid. Chamkha et al. [34] have analyzed the boundary layer analysis for the mixed convection past a vertical wedge in a porous medium saturated with a power law type non-Newtonian nanofluid. Natural convection boundary-layer flow over a permeable vertical cone embedded in a porous medium saturated with a nanofluid in the presence of uniform lateral mass flux was presented by Chamkha et al. [35, 36].

To the best of author knowledge, until now, the flow and heat transfer of dusty fluid past a stretching sheet along with porous media in the presence of nonlinear thermal radiation and non-uniform heat source/sink has never been studied. The numerical solutions are obtained by applying RKF 45 method along with shooting technique.

\section{Mathematical Formulation}

Consider a steady three dimensional boundary layer flow of an incompressible dusty fluid over a horizontal stretching sheet embedded in a porous medium. The sheet is aligned with the $x y$-plane $(z=0)$ and the flow takes place in the domain $z>0$. Let $u_{w}=c \lambda x$ and $v_{w}=$ cy be the velocities of the stretching sheet along the $x-$ and $y$-directions respectively where $c$ is the stretching rate and $\lambda$ is the coefficient which indicates the difference between the sheet velocity components in $x$ and $y$ directions. The particles are taken to be small enough and of sufficient number and are treated as a continuum which allow concepts such as density and velocity to have physical meaning. The dust particles are assumed to be spherical in shape, uniform in size and mass, and are undeformable.

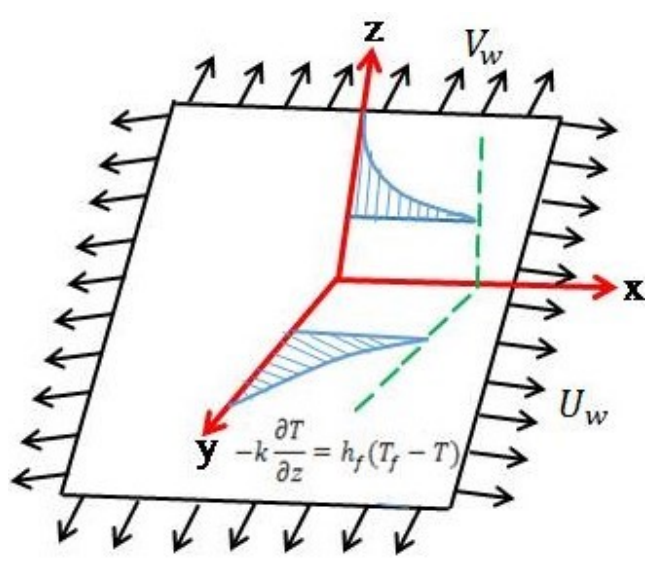

Fig. 1: A schematic flow diagram

The coordinate system and flow regime are illustrated as in Figure 1. The boundary layer equations of three dimensional incompressible dusty fluid are stated as [8];

$$
\begin{gathered}
\frac{\partial u}{\partial x}+\frac{\partial v}{\partial y}+\frac{\partial w}{\partial z}=0 \\
\frac{\partial u}{\partial x}+v \frac{\partial u}{\partial y}+w \frac{\partial u}{\partial z}=v\left(\frac{\partial^{2} u}{\partial x^{2}}+\frac{\partial^{2} u}{\partial y^{2}}+\frac{\partial^{2} u}{\partial z^{2}}\right) \\
+\frac{\rho_{p}}{\rho \tau_{v}}\left(u_{p}-u\right)-\frac{v}{k^{\prime}} u, \\
u \frac{\partial v}{\partial x}+v \frac{\partial v}{\partial y}+w \frac{\partial v}{\partial z}=v\left(\frac{\partial^{2} v}{\partial x^{2}}+\frac{\partial^{2} v}{\partial y^{2}}+\frac{\partial^{2} v}{\partial z^{2}}\right) \\
+\frac{\rho_{p}}{\rho \tau_{v}}\left(v_{p}-v\right)-\frac{v}{k^{\prime}} v, \\
\frac{\partial u_{p}}{\partial x}+\frac{\partial v_{p}}{\partial y}+\frac{\partial w_{p}}{\partial z}=0, \\
u_{p} \frac{\partial u_{p}}{\partial x}+v_{p} \frac{\partial u_{p}}{\partial y}+w_{p} \frac{\partial u_{p}}{\partial z}=\frac{1}{\tau_{v}}\left(u-u_{p}\right), \\
u_{p} \frac{\partial v_{p}}{\partial x}+v_{p} \frac{\partial v_{p}}{\partial y}+w_{p} \frac{\partial v_{p}}{\partial z}=\frac{1}{\tau_{v}}\left(v-v_{p}\right), \\
u_{p} \frac{\partial w_{p}}{\partial x}+v_{p} \frac{\partial w_{p}}{\partial y}+w_{p} \frac{\partial w_{p}}{\partial z}=\frac{1}{\tau_{v}}\left(w-w_{p}\right),
\end{gathered}
$$




$$
\begin{gathered}
u \frac{\partial T}{\partial x}+v \frac{\partial T}{\partial y}+w \frac{\partial T}{\partial z}=\frac{\partial}{\partial z}\left[\left(k+\frac{16 \sigma^{\star} T_{\infty}^{3}}{3 k^{\star}}\right) \frac{\partial T}{\partial z}\right] \frac{1}{\rho c_{p}} \\
+\frac{\rho_{p}}{\rho} \frac{T_{p}-T}{\tau_{T}}+\frac{\rho_{p}}{\rho c_{p}} \frac{1}{\tau_{v}}\left[\left(u_{p}-u\right)^{2}+\left(v_{p}-v\right)^{2}\right]+q^{\prime \prime \prime} \\
u_{p} \frac{\partial T_{p}}{\partial x}+v_{p} \frac{\partial T_{p}}{\partial y}+w_{p} \frac{\partial T_{p}}{\partial z}=\frac{c_{p}}{c_{m}} \frac{T_{p}-T}{\tau_{T}},
\end{gathered}
$$

with boundary conditions as [8];

$$
\begin{aligned}
u & =u_{w}, v=v_{w}, w=0,-k \frac{\partial T}{\partial y}=h_{f}\left(T_{f}-T\right) \text { at } z=0, \\
u_{p} & =u=0, v_{p}=v=0, w_{p}=w, \rho_{p}=\rho \omega, \\
T & =T_{\infty}, \quad T_{p}=T_{\infty} \text { as } z \rightarrow \infty,
\end{aligned}
$$

where $(u, v, w)$ and $\left(u_{p}, v_{p}, w_{p}\right)$ denote the respective velocity components of the fluid and dust phases along the $x, y$ and $z$-directions. $\rho$ and $\rho_{p}$ are the density of the fluid and dust phase respectively. $k, v, c_{p}$ and $c_{m}$ are thermal conductivity, kinematic viscosity, the specific heat of fluid and dust phase, respectively. $\tau_{T}$ is the thermal equilibrium time i.e., the time required by the dust cloud to adjust its temperature to the fluid, $\tau_{v}$ is the relaxation time of the of dust particle i.e., the time required by a dust particle to adjust its velocity relative to the fluid. $T$ and $T_{p}$ represents the temperatures of the fluid and dust particles inside the boundary layer respectively. Throughout the study, it is assumed that, $c_{p}=c_{m}$. In deriving these equations, the drag force is considered for the interaction between the fluid and particle phases. $h_{f}$ is the convective heat transfer coefficient, $T_{f}$ is the convective fluid temperature below the moving sheet. Further, $q^{\prime \prime \prime}$ is the space and temperature dependent internal heat generation/absorption (nonuniform heat source/sink) which can be expressed as;

$$
q^{\prime \prime \prime}=\left(\frac{k u_{w}(x)}{x v}\right)\left[A^{\star}\left(T_{w}-T_{\infty}\right) f^{\prime}(\eta)+B^{\star}\left(T-T_{\infty}\right)\right],
$$

where $A^{\star}$ and $B^{\star}$ are the parameters of the space and temperature dependent internal heat generation/absorption. It is to be noted that, $A^{\star}$ and $B^{\star}$ are positive for internal heat source and negative for internal heat sink.

Introduce the following similarity transformations to reduce the partial differential equations in to set of ordinary ones;

$$
\begin{gathered}
u=c \lambda x f^{\prime}(\eta), v=c y\left[f^{\prime}(\eta)+g^{\prime}(\eta)\right], \\
w=-\sqrt{c v}[g(\eta)+(\lambda+1) f(\eta)], \eta=\sqrt{\frac{c}{v}} z, \\
u_{p}=c \lambda x F(\eta), v_{p}=c y[F(\eta)+G(\eta)], \\
w_{p}=\sqrt{c v}[G(\eta)+(\lambda+1) K(\eta)],
\end{gathered}
$$

$$
\rho_{r}=\frac{\rho_{p}}{\rho}=H(\eta)
$$

Here prime denotes differentiation with respect to $\eta$. Making use of (12) in equations (1) to (7), continuity equations (1) and (4) are identically satisfied and the remaining momentum equations take the following form:

$$
\begin{gathered}
f^{\prime \prime \prime}+[g+(\lambda+1) f] f^{\prime \prime}-f^{\prime} 2 \lambda+\beta\left(F-f^{\prime}\right) H-k_{p} f^{\prime}=0, \\
\left(f^{\prime \prime \prime}+g^{\prime \prime \prime}\right)+[g+(\lambda+1) f]\left(f^{\prime \prime}+g^{\prime \prime}\right)-\left(f^{\prime}+g^{\prime}\right)^{2}+ \\
\beta\left[(F+G)-\left(f^{\prime}+g^{\prime}\right)\right]-k_{p}\left[f^{\prime}+g^{\prime}\right]=0, \\
{[G+(\lambda+1) K] F^{\prime}+\lambda F^{2}+\beta\left(F-f^{\prime}\right)=0,} \\
{[F+G]^{2}+[G+(\lambda+1) K]\left[F^{\prime}+G^{\prime}\right]} \\
+\beta\left[(F+G)-\left(f^{\prime}+g^{\prime}\right)\right]=0, \\
{[G+(\lambda+1) K]\left[(\lambda+1) K^{\prime}+G^{\prime}\right]} \\
+\beta[G+g+(\lambda+1)(K+f)]=0,
\end{gathered}
$$

$[G+(\lambda+1) K] H^{\prime}+\left[(\lambda+1)\left(F+K^{\prime}\right)+G+G^{\prime}\right] H=0$.

Corresponding boundary conditions becomes;

At $\eta=0: f^{\prime}=1, f=0, g=g^{\prime}=0$.

A $\eta \rightarrow \infty: f^{\prime}=F=0, g^{\prime}=G=0, K=-f-\frac{g}{\lambda+1}, H=\omega$.

where, $\beta=1 / c \tau_{v}$ is the fluid-particle interaction parameter, $H=\rho_{p} / \rho$ is the relative density and $k_{p}=\frac{v}{k^{\prime} c}$ is the permeability parameter and $\omega$ is the density ratio and is considered as 0.2 in this present study.

\subsection{Heat transfer solution}

To transform the energy equations into a non-dimensional form, dimensionless temperature profile for the clean fluid and dusty fluid are introduced as follows:

$$
\theta(\eta)=\frac{T-T_{\infty}}{T_{f}-T_{\infty}}, \theta_{p}(\eta)=\frac{T_{P}-T_{\infty}}{T_{f}-T_{\infty}}
$$

where, $T_{\infty}$ denotes the temperature at larger distance from the wall with $T=T_{\infty}\left(1+\left(\theta_{w}-1\right) \theta\right)$ and $\theta_{w}=\frac{T_{f}}{T_{\infty}}$ being the temperature ratio parameter. Making use of equations (12) and (20) in equations (8) and (9) the energy equations takes the following form:

$$
\left(\left(1+R d\left(1+\left(\theta_{w}-1\right) \theta\right)^{3}\right) \theta^{\prime}\right)^{\prime}+\operatorname{Pr}[g+(\lambda+1) f] \theta^{\prime}
$$




$$
\begin{aligned}
& +\operatorname{Pr} \beta_{\tau}\left[\theta_{p}-\theta\right] H+\operatorname{Pr} \beta\left[E c_{X}\left(F-f^{\prime}\right)^{2}\right. \\
& \left.+E c_{y}\left(F-f^{\prime}+G-g^{\prime}\right)^{2}\right] H+\lambda\left(A^{\star} f^{\prime}+B^{\star} \theta\right)=0, \\
& {[G+(\lambda+1)+K] \theta_{p}^{\prime}+\frac{c_{p}}{c_{m}} \beta_{\tau}\left[\theta_{p}-\theta\right]=0 .}
\end{aligned}
$$

where, $R d=\frac{16 \sigma^{\star} T_{\infty}^{3}}{3 k k^{\star}}$ is the radiation parameter, $\operatorname{Pr}=\frac{\mu c_{p}}{k}$ is the Prandtl number, $E c_{x}=\frac{\left(u_{w}\right)^{2}}{\left(T_{f}-T_{\infty}\right) c_{p}}$ and $E c_{y}=\frac{\left(v_{w}\right)^{2}}{\left(T_{f}-T_{\infty}\right) c_{p}}$ are the Eckert numbers and $\beta_{\tau}=\frac{1}{\tau_{T} c}$ is the fluid-particle interaction parameter for temperature. The corresponding boundary conditions take the following form;

$$
\begin{gathered}
\eta=0: \theta^{\prime}=-B_{i}(1-\theta), \\
\eta \rightarrow \infty: \theta_{p}=\theta=0 .
\end{gathered}
$$

where $B_{i}=\sqrt{\frac{v}{c}} \frac{h_{f}}{k}$ is the Biot number. The wall shear stress is given by,

$$
\tau_{z x}=-\mu\left(\frac{\partial u}{\partial z}+\frac{\partial w}{\partial x}\right)_{z=0}, \tau_{z y}=-\mu\left(\frac{\partial v}{\partial z}+\frac{\partial w}{\partial y}\right)_{z=0}
$$

The friction factor is written as,

$$
C f_{X} \operatorname{Re}_{x}^{\frac{1}{2}}=-f^{\prime \prime}(0), C f_{y} \operatorname{Re}_{y}^{\frac{1}{2}}=-g^{\prime \prime}(0)
$$

The surface heat transfer rate is given by,

$$
q_{w}=-k\left(\frac{\partial T}{\partial y}\right)_{z=0}+\left(q_{r}\right)_{w}
$$

The local Nusselt number is written as

$$
N u R e_{x}^{-\frac{1}{2}}=-\left(1+R d \theta_{w}^{3}\right) \theta^{\prime}(0)
$$

\section{Numerical solution}

The set of non-linear differential equations (13-18) and (21-22) with boundary conditions (19) and (23) have been solved using Runge-Kutta-Fehlberg fourth-fifth order method along with shooting technique. In the first step, equations of higher order are discretized to a system of simultaneous differential equations of first order by introducing new dependent variables. Missed initial conditions are obtained with the help of shooting technique. Afterward, a finite value for $\eta_{\infty}$ is chosen in such a way that all the far field boundary conditions are satisfied asymptotically. Our bulk computations are considered with the value at $\eta_{\infty}=5$, which is sufficient to achieve the far field boundary conditions asymptotically for all values of the parameters considered.

\section{Results and discussion}

Numerical computation has been carried out in order to study the influence of different parameters that describes the flow and heat transfer characteristics of dusty fluid. The results are presented and discussed in detail through graphical representation and tables.

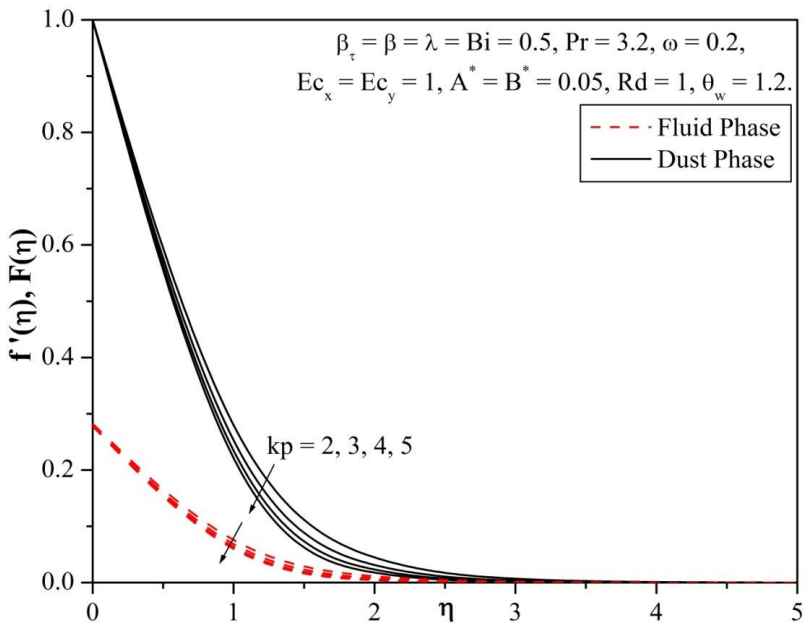

Fig. 2: Dimensionless profiles of $u, u_{p}$ velocity components for different values of $k_{p}$.

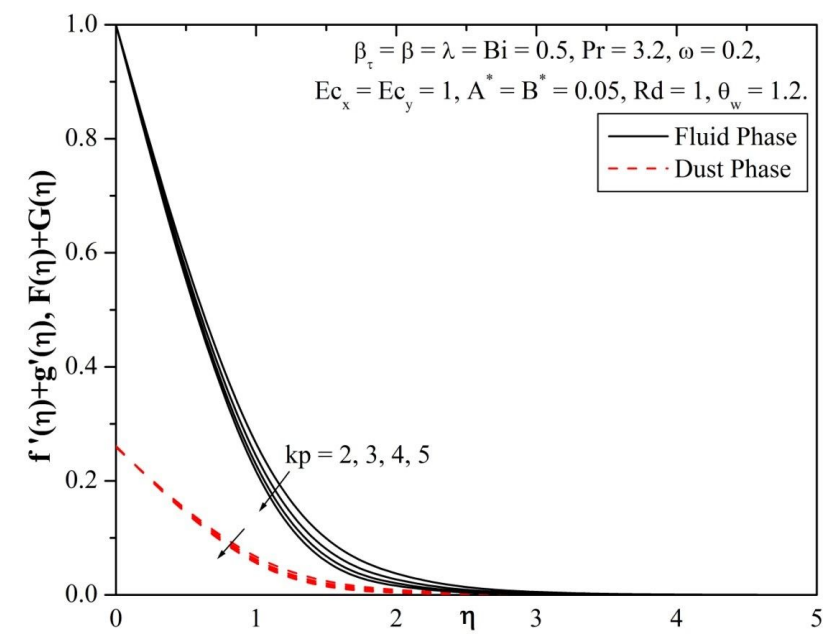

Fig. 3: Dimensionless profiles of $v, v_{p}$ velocity components for different values of $k_{p}$.

The dimensionless velocity profiles for different values of $k_{p}$ proportional to $u$ and $v$ velocity components are depicted in Figure 2 and 3, respectively for both fluid and dust phase. Here $f^{\prime}(\eta)$ represents the velocity in $x$-direction while $\left[f^{\prime}(\eta)+g^{\prime}(\eta)\right]$ is the velocity along 


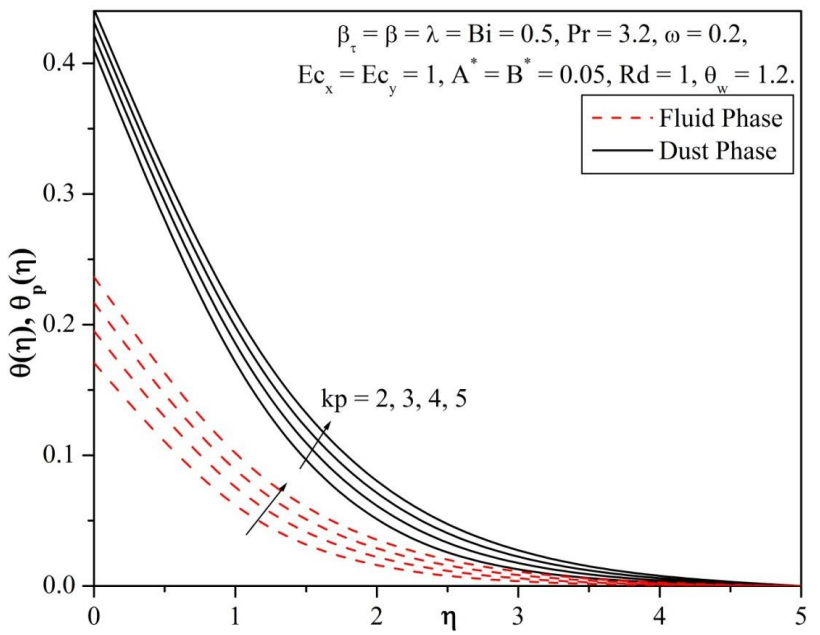

Fig. 4: Dimensionless temperature profiles for different values of $k_{p}$.

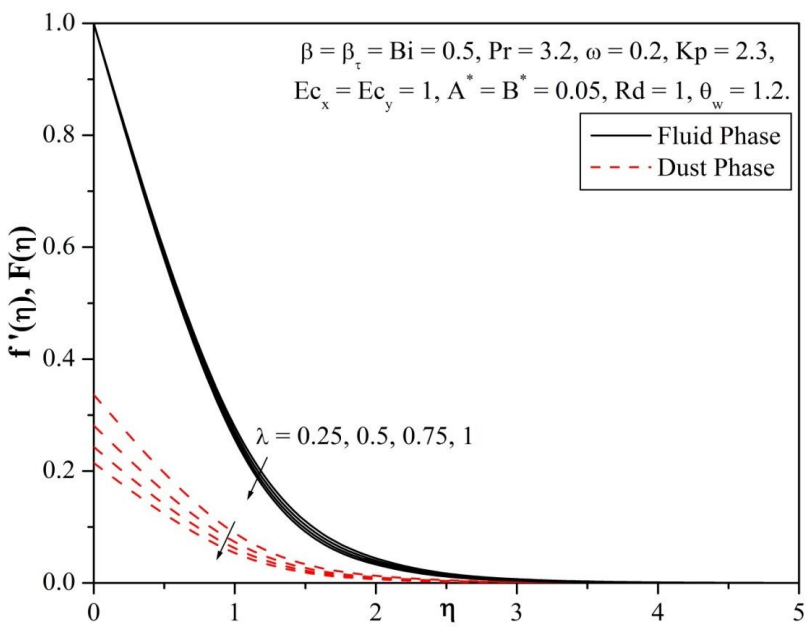

Fig. 5: Dimensionless profiles of $u, u_{p}$ velocity components for different values of $\lambda$.

$y$-direction for fluid phase. Further, $F(\eta)$ represents the velocity in $x$-direction while $[F(\eta)+G(\eta)]$ is the velocity in $y$-direction for dust phase respectively. From these figures, it is observed that both the velocities are retarded for the case of increasing $k_{p}$. This is because, an increase in permeability parameter causes the resistance to the fluid motion and hence velocity decreases for both fluid and dust phase. The effect of increasing values of permeable parameter contributes to the thickening of thermal boundary layer, and is shown in Figure 4. This is evident from the fact that, the porous medium opposes the fluid motion. The resistance offered to the flow is responsible in enhancing the temperature.

The effect of various values of $\lambda$ for dimensionless velocity profiles are depicted in Figure 5 and 6 for both fluid and dust phase. These figures portraits that, the behavior

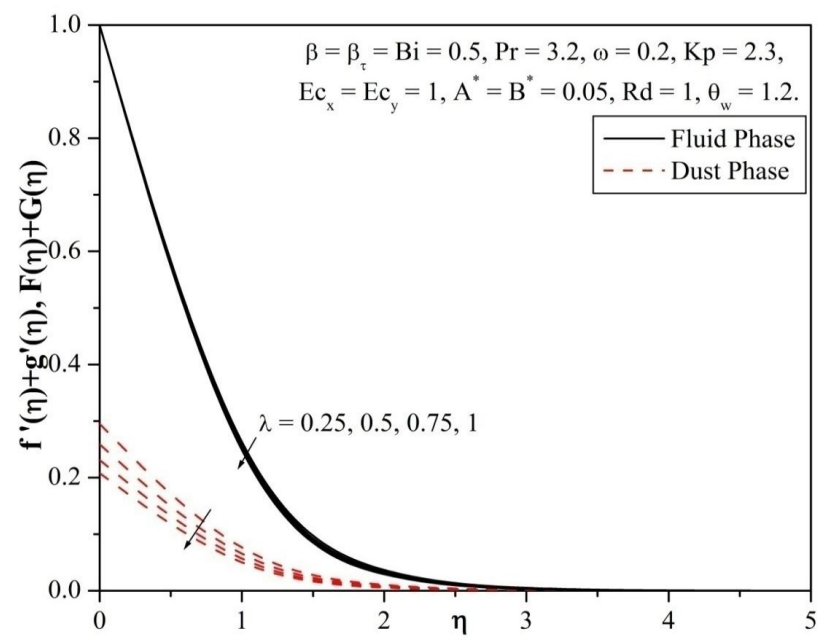

Fig. 6: Dimensionless profiles of $v, v_{p}$ velocity components for different values of $\lambda$.

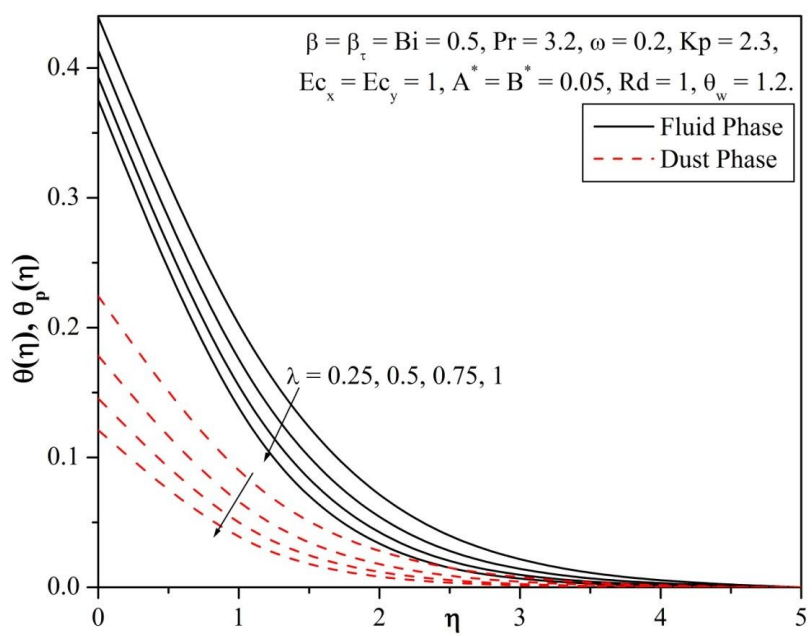

Fig. 7: Dimensionless temperature profiles for different values of $\lambda$.

of the $f^{\prime}(\eta), F(\eta), f^{\prime}(\eta)+g^{\prime}(\eta)$ and $F(\eta)+G(\eta)$ are the same and it decreases with increase in values of $\lambda$. It can also be seen that, fluid phase velocity is greater than dust phase velocity. The variation of dimensionless temperature profile for both phases along with different values of $\lambda$, are illustrated in Figure 7. From this figure it is seen that, increase of $\lambda$ causes decrease of the temperature profile of both dusty and fluid phase. Furthermore, one can observe from this figure that values of the temperature are higher for clean fluid than for the dusty fluid at all points, as excepted.

Figures 8 and 9 are plotted to view the effect of fluid particle interaction parameter $\beta$ on velocity profile respectively in both directions for fluid and dust phase. From these figures it is examined that, the velocity $u, v$ decays while $u_{p}, v_{p}$ is enhanced for larger values of $\beta$. 


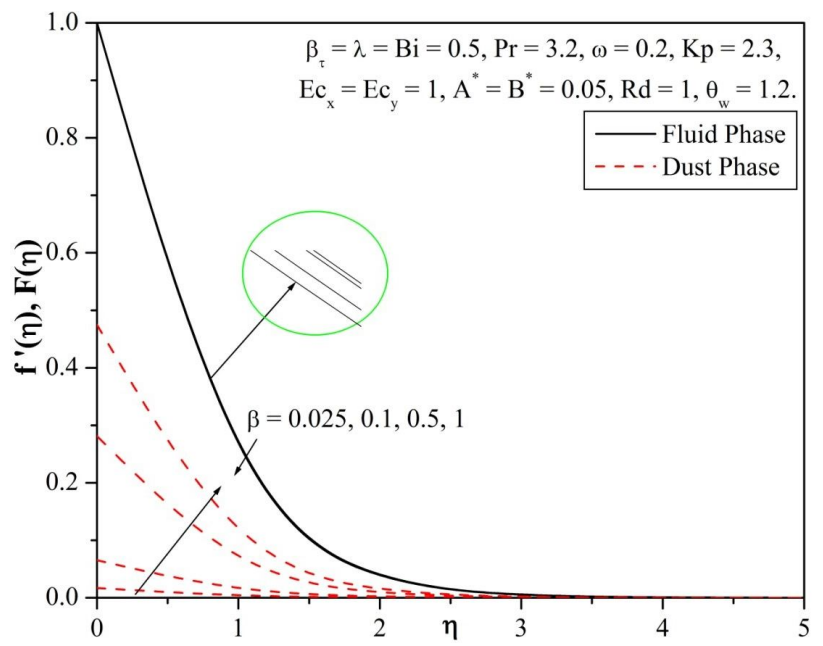

Fig. 8: Dimensionless profiles of $u, u_{p}$ velocity components for different values of $\beta$.

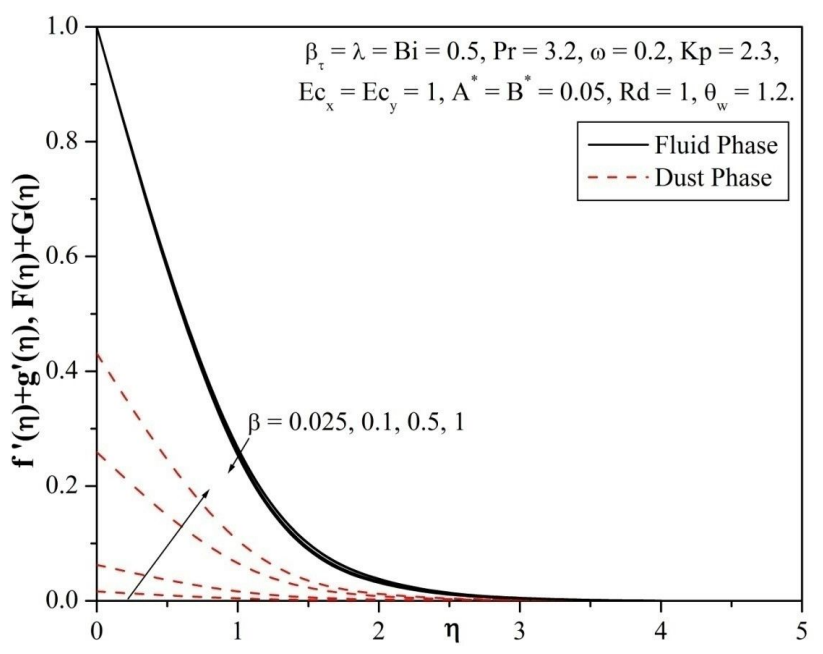

Fig. 9: Dimensionless profiles of $v, v_{p}$ velocity components for different values of $\beta$.

The variation of dimensionless temperature profiles for different values of space-dependent heat source/sink parameter $A^{*}$ and temperature-dependent heat source/sink parameter $B^{\star}$ are plotted in Figures 10 and 11 respectively. It is evident from these graphs that, increasing $A^{\star}$ and $B^{\star}$ results in the enhancement of both fluid phase and dust phase temperature.

Figure 12 and 13 characterize the temperature profiles for distinct values of fluid and thermal particle interaction parameter $\beta$ and $\beta_{\tau}$ respectively. Figure 12 indicates that the temperature of both phases increases with increases in $\beta$ and it reveals that, effect of variation of $\beta$ is more sensible on dusty phase than for the fluid phase. This is because of the direct effect of $\beta$ on velocity and since the temperature depends on velocity then the temperature varies with

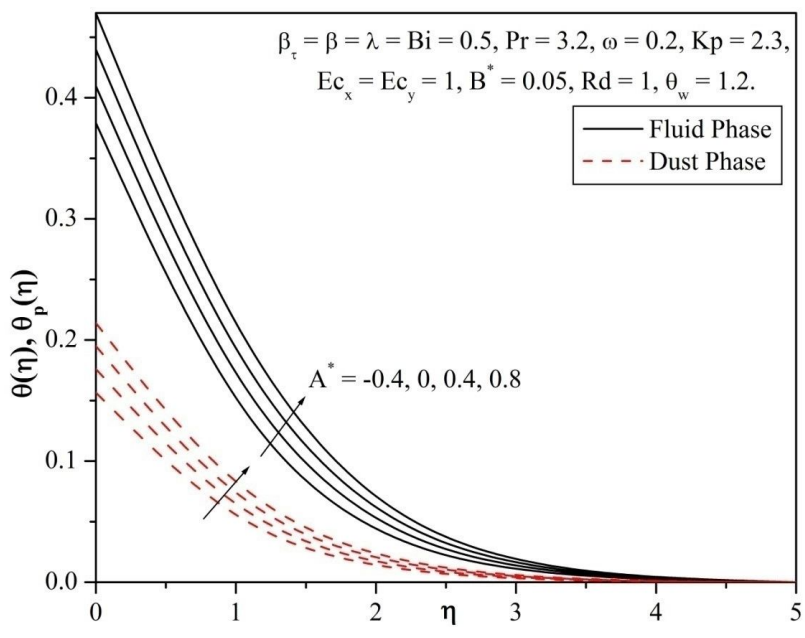

Fig. 10: Dimensionless temperature profiles for different values of $A^{*}$.

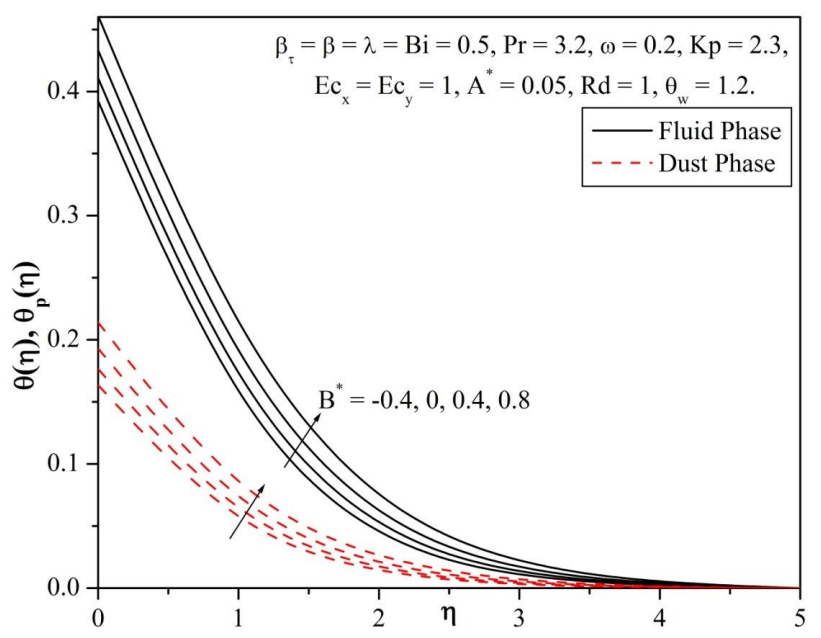

Fig. 11: Dimensionless temperature profiles for different values of $B^{\star}$.

variation of $\beta$. Effect of $\beta_{\tau}$ on fluid and dust phase temperature profiles for both linear and nonlinear thermal radiation cases are shown in Figure 13. It can be seen that fluid phase temperature decreases and dust phase temperature increases with increase in $\beta_{\tau}$ for both the cases. It can also be seen that nonlinear radiation is more influential than linear radiation.

Figures 14 and 15 illustrate the effect of the Eckert number $(E c)$ on temperature distribution. These figures show that the increasing values of Eckert number is to increase the temperature distribution. This is due to the fact that, heat energy is stored in the liquid due to frictional heating which results into increasing in its temperature and this is true in both the cases.

Figure 16 resemble the change in temperature profile for different values of Biot number (Bi). It describes that 


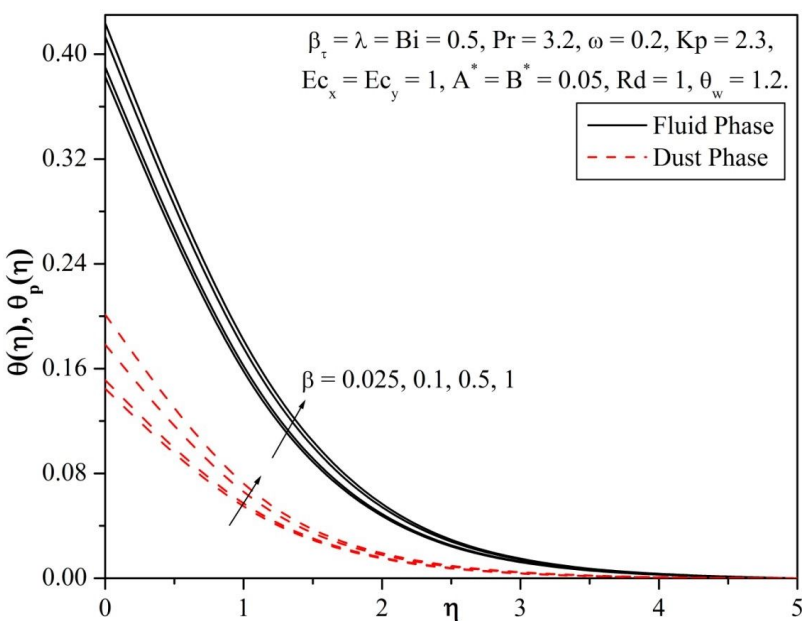

Fig. 12: Dimensionless temperature profiles for different values of $\beta$.
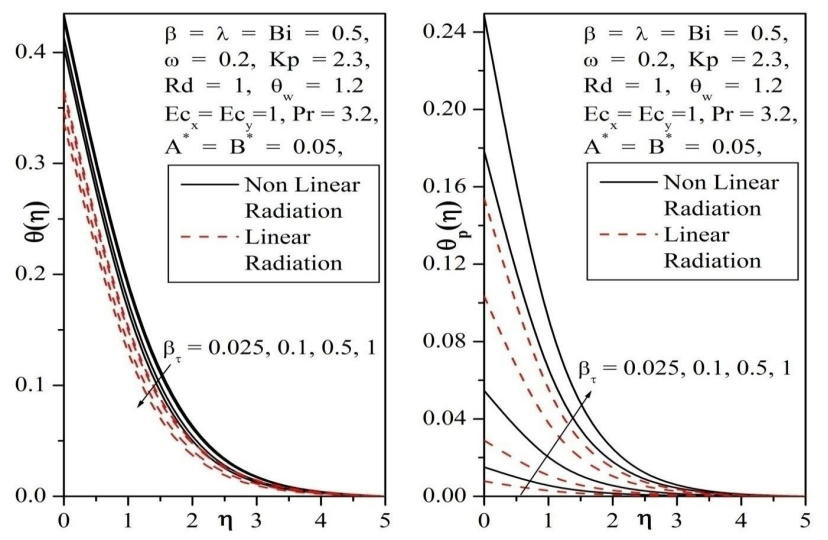

Fig. 13: Dimensionless temperature profiles for different values of $\beta_{\tau}$.

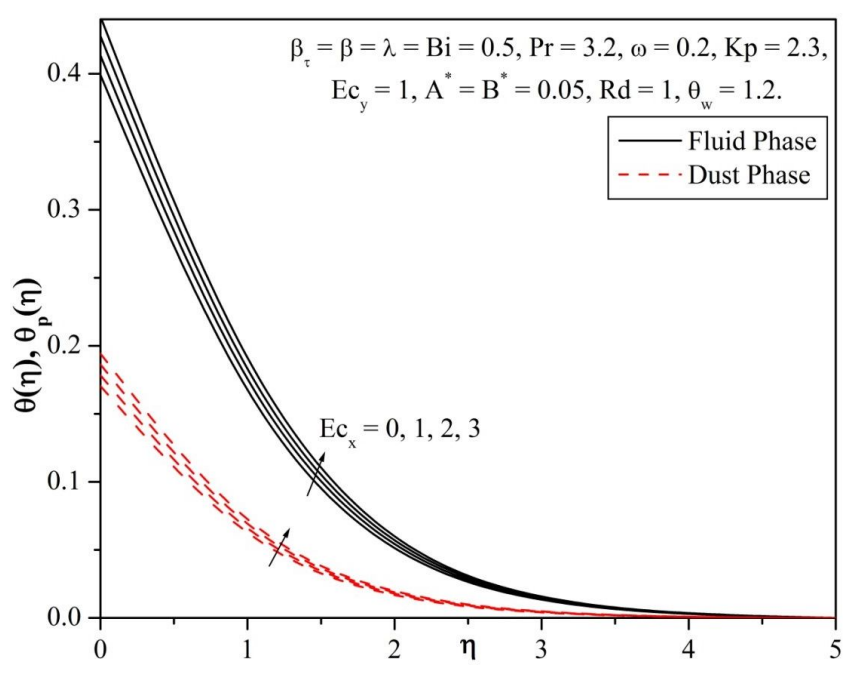

Fig. 14: Dimensionless temperature profiles for different values of $E c_{x}$.

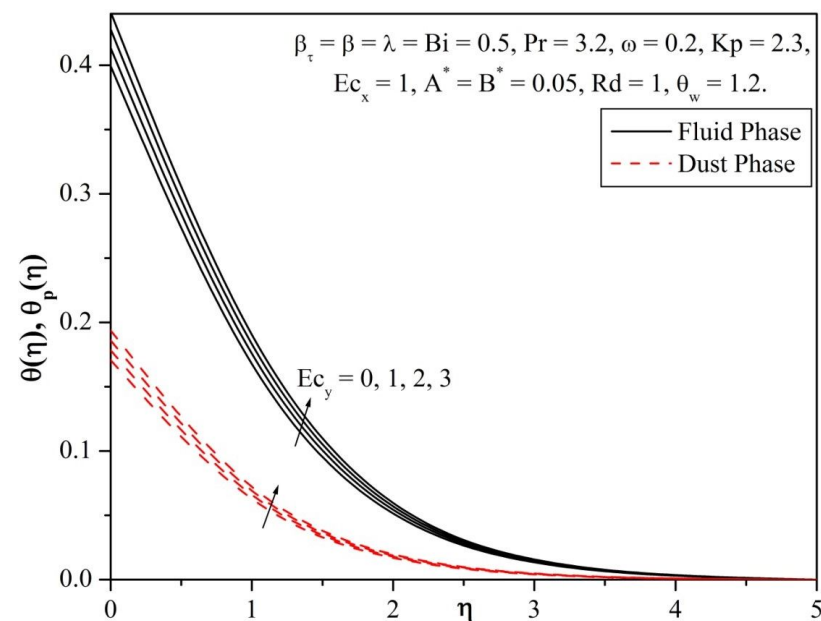

Fig. 15: Dimensionless temperature profiles for different values of $E c_{y}$.

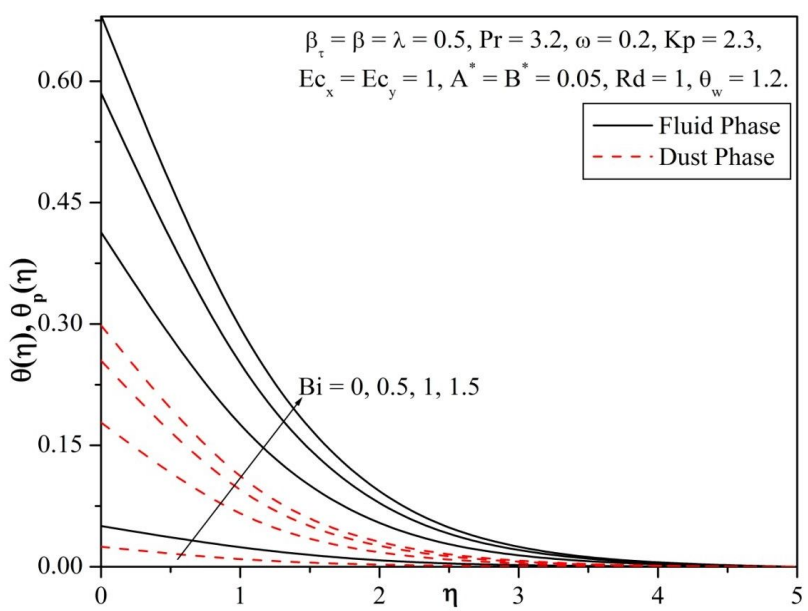

Fig. 16: Dimensionless temperature profiles for different values of $B_{i}$.
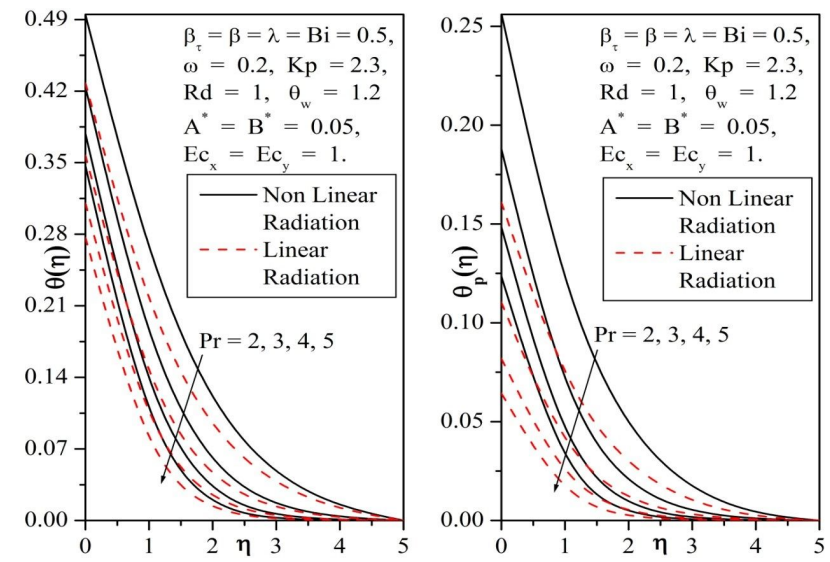

Fig. 17: Dimensionless temperature profiles for different values of $\mathrm{Pr}$. 

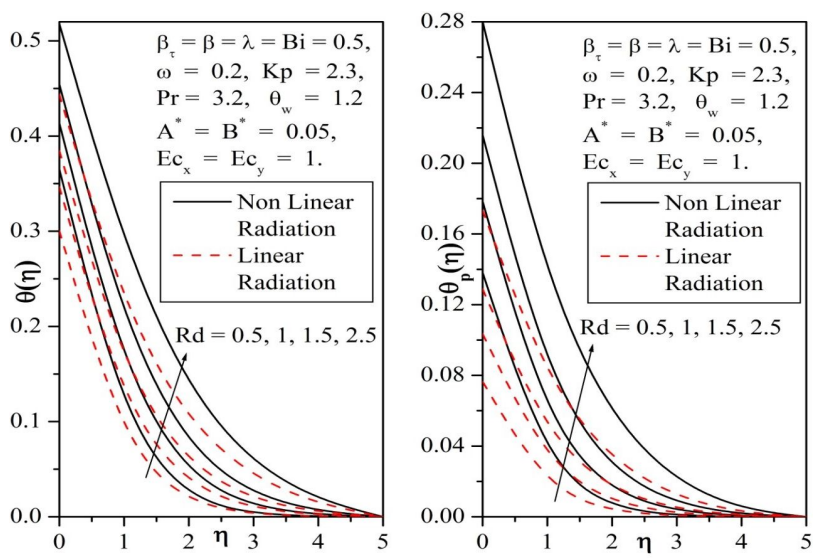

Fig. 18: Dimensionless temperature profiles for different values of $R d$.

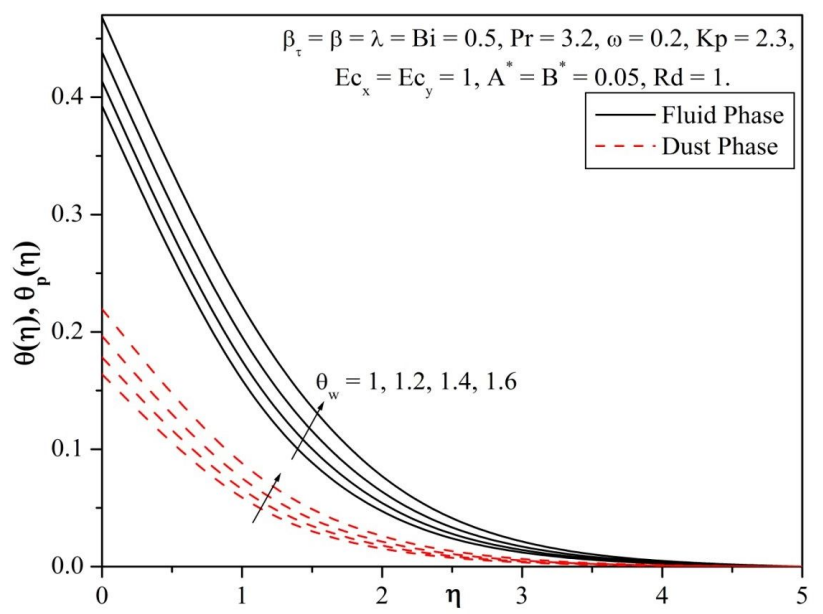

Fig. 19: Dimensionless temperature profiles for different values of $\theta_{w}$.

the increasing values of Biot number lead to elevated temperature and thicker thermal boundary layer. This is due to the fact that, the convective heat exchange at the surface leads to enhance the thermal boundary layer thickness.

Figure 17 is sketched for the temperature distribution against the Prandtl number $(\mathrm{Pr})$. From this figure, it reveals that the temperature decreases with increase in the value of $P r$. This is because, as the Prandtl number increases, thermal diffusivity decreases there by decreases the temperature. Hence Prandtl number can be used to increase the rate of cooling. Figure 18 exhibits the variation of temperature profile for the radiation parameter. As, the radiation parameter releases the heat energy into the flow, with an increase of radiation parameter, temperature profile increases.

The effect of temperature ratio parameter $\left(\theta_{w}\right)$ over the dimensionless temperature is shown in Figure 19. It is observed that, the temperature profile increases for both phases for the increasing the values of $\theta_{w}$. This is because, fluid temperature is much higher than the ambient temperature for increasing values of $\theta_{w}$, which increases the thermal state of the fluid. From the obtained result it can be concluded that, non linear radiation has more influence as compared to linear radiation.

Table 1: Values of skin friction coefficient and Nusselt number for $K_{p}$ and $\beta$.

\begin{tabular}{ccccc}
\hline $\boldsymbol{K}_{\boldsymbol{p}}$ & $\boldsymbol{\beta}$ & $\boldsymbol{C f}_{\boldsymbol{x}} \boldsymbol{R e}_{\boldsymbol{x}}^{\frac{1}{2}}$ & $\boldsymbol{C f}_{\boldsymbol{y}} \boldsymbol{R e}_{\boldsymbol{y}}^{\frac{1}{2}}$ & $\boldsymbol{N u R e}_{\boldsymbol{x}}^{-\frac{1}{2}}$ \\
\hline $\mathbf{2}$ & & 1.80414 & 2.48579 & 0.868129 \\
$\mathbf{3}$ & & 2.06244 & 2.66918 & 0.851712 \\
$\mathbf{4}$ & & 2.29192 & 2.84405 & 0.836969 \\
& $\mathbf{0 . 1}$ & 1.87066 & 2.40828 & 0.850894 \\
& $\mathbf{0 . 4}$ & 1.88209 & 2.51217 & 0.860442 \\
& $\mathbf{0 . 7}$ & 1.89111 & 2.59441 & 0.867504 \\
\hline
\end{tabular}

Table 2: Values of Nusselt number for various existing parameters

\begin{tabular}{|c|c|c|c|c|c|c|c|c|c|}
\hline$A^{\star}$ & $B^{\star}$ & $B i$ & $E c_{x}$ & $E c_{y}$ & $\mathrm{Pr}$ & $R d$ & $\theta_{w}$ & $\boldsymbol{\beta}_{\tau}$ & $N u R e_{x}^{-\frac{1}{2}}$ \\
\hline 0 & 0.05 & 0.5 & 1 & 1 & 3.2 & 1 & 1.2 & 0.5 & 0.864929 \\
\hline 0.4 & & & & & & & & & 0.849573 \\
\hline \multirow[t]{25}{*}{0.8} & & & & & & & & & 0.834262 \\
\hline & 0 & & & & & & & & 0.863836 \\
\hline & 0.4 & & & & & & & & 0.857119 \\
\hline & 0.8 & & & & & & & & 0.32066 \\
\hline & & 0.5 & & & & & & & 0.863007 \\
\hline & & 1 & & & & & & & 1.232436 \\
\hline & & 1.5 & & & & & & & 1.426802 \\
\hline & & & 0 & & & & & & 0.863576 \\
\hline & & & 0.5 & & & & & & 0.863292 \\
\hline & & & 1.5 & & & & & & 0.862723 \\
\hline & & & & 0 & & & & & 0.865188 \\
\hline & & & & 0.5 & & & & & 0.864098 \\
\hline & & & & 1.5 & & & & & 0.861915 \\
\hline & & & & & 3 & & & & 0.846785 \\
\hline & & & & & 4 & & & & 0.916951 \\
\hline & & & & & 5 & & & & 0.966899 \\
\hline & & & & & & 0.5 & & & 0.641931 \\
\hline & & & & & & 1.5 & & & 1.052475 \\
\hline & & & & & & 2.5 & & & 1.368408 \\
\hline & & & & & & & 1 & & 0.653644 \\
\hline & & & & & & & 1.4 & & 1.137426 \\
\hline & & & & & & & 1.6 & & 1.471094 \\
\hline & & & & & & & & 0.1 & 0.839874 \\
\hline & & & & & & & & 0.4 & 0.858548 \\
\hline & & & & & & & & 0.7 & 0.870296 \\
\hline
\end{tabular}

Variations of skin friction coefficient and local Nusselt number for the governing parameters are recorded in the Table 1 and 2. From these table, it is clear that skin friction coefficient in $x$ and $y$ direction increases with $K_{p}$ and $\beta$. Further, Nusselt number decreases for $K_{p}$ and increases 
for $\beta$. Moreover, Nusselt number are increasing functions of $\operatorname{Pr}, \beta_{T}, B_{i}$ while it decreases for $A^{\star}, B^{\star}, R d, \theta_{w}$ and $E c$.

\section{Conclusion}

In the present study, three-dimensional boundary layer flow and heat transfer of a dusty fluid towards a stretching sheet embedded in a porous medium in presence of non-uniform source/sink with convective boundary condition is investigated. By using the appropriate transformation for the velocity and temperature, the basic equations governing the flow and heat transfer were reduced to a set of ordinary differential equations. These equations are solved numerically using the fourth-fifth-order RungeKutta-Fehlberg method. Some of the conclusion obtained from this investigation are summarized as follows:

1. Fluid phase velocity is always greater than that of the particle phase.

2. Velocity of fluid and dust phases decrease with increases in permeability parameter.

3. Velocity and temperature profile of fluid phase and dust phases decrease with increases in velocity ratio parameter $\lambda$.

4. Increase of $\beta$ will decrease fluid phase velocity and increases dust phase velocity.

5. Increase of $\beta_{T}$ will decrease fluid phase and increases the dust phase of temperature profile.

6. Fluid phase temperature is higher than the dust phase temperature.

7. Temperature profiles of fluid and dust phases increases with the increase of the $E c, A^{\star}, B^{\star}, B i, R d$ and $\theta_{w}$.

\section{References}

[1] Saffman, P.G., On the stability of laminar flow of a dusty gas, Journal of Fluid Mechanics, 13, 120-128 (1962)

[2] Vajravelu, K. and Nayfeh, J., Hydromagnetic Flow of a Dusty Fluid over a Stretching Sheet, Int. J. of Nonlinear Mechanics, 27, 937-945 (1992)

[3] Gireesha, B. J., Ramesh, G. K., Abel, M. S., and Bagewadi, C. S., Boundary layer flow and heat transfer of a dusty fluid flow over a stretching sheet with non-uniform heat source/sink, International Journal of Multiphase Flow, 37, 977-982 (2011)

[4] Manjunatha, P. T., Gireesha, B. J., and Prasannakumara, B. C., Thermal analysis of conducting dusty fluid flow in a porous medium over a stretching cylinder in the presence of nonuniform source/sink, International Journal of Mechanical and Materials Engineering, 1(13), 10 Pages (2014)
[5] Prakash, O. M., Makinde, O. D., Kumar, D., and Dwivedi, Y. K., Heat transfer to MHD oscillatory dusty fluid flow in a channel filled with a porous medium, Sadhana, 40(4), 1273-1282 (2015)

[6] Attia, H. A., Abbas, W., El-Din Abdin, A., and Abdeen, M. A. M., Effects of ion slip and hall current on unsteady couette flow of a dusty fluid through porous media with heat transfer, High Temperature, 53(6), 891-898 (2015)

[7] Muthuraj, R., Nirmala, K., and Srinivas, S., Influences of chemical reaction and wall properties on MHD Peristaltic transport of a Dusty fluid with Heat and Mass transfer, Alexandria Engineering Journal, 55, 597-611 (2016)

[8] Mohaghegh, M. R. and Rahimi, A. B., Three-dimensional Stagnation-point flow and heat transfer of a dusty fluid toward a stretching sheet, Journal of Heat Transfer, 138(11), 112001 (2016)

[9] Mahanthesh, B., Gireesha, B. J., PrasannaKumara, B. C., and Shashikumar, N. S., Marangoni convection radiative flow of dusty nanoliquid with exponential space dependent heat source, Nuclear Engineering and Technology, 49(8), 16601668 (2017).

[10] Al-Rashed, Siddiqa, S., Begum, N., and Anwar Hossain, Md., Numerical solutions for a compressible dusty fluid flow along a vertical wavy cone, International Journal of Heat and Mass Transfer, 108, 1229-1236 (2017)

[11] Gireesha, B. J., Venkatesh, P., Shashikumar, N. S., and Prasannakumara, B. C. Boundary layer flow of dusty fluid over a radiating stretching surface embedded in a thermally stratified porous medium in the presence of uniform heat source, Nonlinear Engineering, 6(1), 31-41 (2017).

[12] Chamkha, A. J., Solar radiation assisted natural convection in uniform porous medium supported by a vertical flat plate. Journal of heat transfer, 119(1), 89-96 (1997).

[13] Chamkha, A. J., Issa, C., and Khanafer, K., Natural convection from an inclined plate embedded in a variable porosity porous medium due to solar radiation, International Journal of Thermal Sciences, 41(1), 73-81 (2002).

[14] Chamkha, A. J., Abbasbandy, S., Rashad, A. M., and Vajravelu, K., Radiation effects on mixed convection over a wedge embedded in a porous medium filled with a nanofluid, Transport in Porous Media, 91(1), 261-279 (2012).

[15] Chamkha, A. J., Abbasbandy, S., Rashad, A. M., and Vajravelu, K., Radiation effects on mixed convection about a cone embedded in a porous medium filled with a nanofluid, Meccanica, 48(2), 275-285 (2013).

[16] Prasannakumara, B. C., and Shashikumar, N. S., Boundary Layer Flow and Heat Transfer of Nanofluid with Fluid Particle Suspension Over a Nonlinear Stretching Sheet in the Presence of Thermal Radiation, Journal of Nanofluids, 6(3), 487-495 (2017).

[17] Mahanthesh, B., Gireesha, B. J., Shashikumar, N. S., Hayat, T., \& Alsaedi, A. Marangoni convection in Casson liquid flow due to an infinite disk with exponential space dependent heat source and cross-diffusion effects, Results in Physics, 9, 78-85 (2018).

[18] Mahanthesh, B., Gireesha, B. J., Shashikumar, N. S., and Shehzad, S. A. Marangoni convective MHD flow of SWCNT and MWCNT nanoliquids due to a disk with solar radiation and irregular heat source, Physica E: Low-dimensional Systems and Nanostructures, 94, 25-30 (2017). 
[19] Hayat, T., Qayyum, S., Alsaedi, A., and Shehzad, S. A., Nonlinear thermal radiation aspects in stagnation point flow of tangent hyperbolic nanofluid with double diffusive convection, Journal of Molecular Liquids, 223, 969-978 (2016)

[20] Shehzad S.A., Hayat T., Alsaedi A. and Mustafa A.O., Nonlinear thermal radiation in three-dimensional flow of Jeffrey nanofluid: A model for solar energy, Appl. Mathe. and Compu., 248, 273-286 (2014)

[21] Hayat T., Muhammad T., Alsaedi A. and Alhuthali M.S., Magnetohydrodynamic three-dimensional flow of viscoelastic nanofluid in the presence of nonlinear thermal radiation, J. Magnetism and Magnetic Materials, 385, 222-229 (2015)

[22] Makinde, O. D., and Animasaun. L., Thermophoresis and Brownian motion effects on MHD bioconvection of nanofluid with nonlinear thermal radiation and quartic chemical reaction past an upper horizontal surface of a paraboloid of revolution, Journal of Molecular Liquids, 221, 733-743 (2016)

[23] Shashikumar, N. S., Archana, M., Prasannakumara, B. C., Gireesha, B. J., and Makinde, O. D., Effects of Nonlinear Thermal Radiation and Second Order Slip on Casson Nanofluid Flow between Parallel Plates, In Defect and Diffusion Forum, 377, 84-94 (2017).

[24] Prasannakumara, B. C., Shashikumar, N. S., and Venkatesh, P., Boundary Layer Flow and Heat Transfer of fluid particle suspension with nanoparticles over a nonlinear stretching sheet embedded in a porous medium, Nonlinear Engineering, 6(3), 179-190 (2017).

[25] Wang C.Y., The three-dimensional flow due to a stretching sheet, Physics of Fluids, 27, 1915-1917 (1984).

[26] Takhar H. S., Chamkha A. J. and Nath G, Unsteady threedimensional MHD-boundary-layer flow due to the impulsive motion of a stretching surface, Acta Mechanica, 146(1-2), 59-71 (2001).

[27] Ahmad I., Ahmad M., Abbas A. and Sajid M., Hydromagnetic flow and heat transfer over a bidirectional stretching surface in a porous medium, Therm. Sci., 15, 205-220 (2011).

[28] Ahmad K. and Nazar R., Magnetohydrodynamic three dimensional flow and heat transfer over a stretching surface in a viscoelastic fluid, Journal of science and technology, 3(1), 1-14 (2011).
[29] Choudhury R. and Das U.J., Viscoelastic effects on free convective three-dimensional flow with heat and mass transfer, ISRN Computational Mathematics, 2012 (2012) http://dx.doi.org/10.5402/2012/402037.

[30] Nadeem S., Rizwan UI Haq, Akbar N.S. and Khan Z.H., MHD three-dimensional Casson fluid flow past a porous linearly stretching sheet, Alexandria Engineering Journal, 52(4), 577582 (2013)

[31] Ramzan M.M., Farooq M., Alsaedi A. and Hayat T., MHD three dimensional flow of couple stress fluid with, Eur. Phys. J. Plus, 128(5), 49-63 (2013).

[32] Chamkha, A., Gorla, R. S. R. and Ghodeswar, K., Non-similar solution for natural convective boundary layer flow over a sphere embedded in a porous medium saturated with a nanofluid, Transport in Porous Media, 86(1), 13-22 (2011).

[33] Gorla, R. S. R. and Chamkha, A., Natural convective boundary layer flow over a horizontal plate embedded in a porous medium saturated with a nanofluid, Journal of Modern Physics, 2(2), 62 (2011).

[34] Chamkha, A. J., Rashad, M. and Subba Reddy Gorla, R., Nonsimilar solutions for mixed convection along a wedge embedded in a porous medium saturated by a non-Newtonian nanofluid: Natural convection dominated regime, International Journal of Numerical Methods for Heat \& Fluid Flow, 24(7), 1471-1486 (2014).

[35] Chamkha, A. J. and Rashad, A. M., Natural convection from a vertical permeable cone in a nanofluid saturated porous media for uniform heat and nanoparticles volume fraction fluxes, International Journal of Numerical Methods for Heat \& Fluid Flow, 22(8), 1073-1085 (2012).

[36] Chamkha, A. J, Abbasbandy, S. and Rashad, A. M., Non-Darcy natural convection flow for non-Newtonian nanofluid over cone saturated in porous medium with uniform heat and volume fraction fluxes, International Journal of Numerical Methods for Heat \& Fluid Flow, 25(2), $422-437$ (2015). 